\title{
Industrialización integral de la alcachofa en pasta nutricional y para alimentos balanceados
}

Recepción: Febrero de 2008 / Aceptación: Mayo de 2008

${ }^{(1)}$ Teonila García Zapata

\section{RESUMEN}

Se realizó un estudio de tipo descriptivo y experimental, con el objeto de procesar la alcachofa para la obtención de pasta, tanto para la alimentación humana como para uso medicinal y artículos de belleza, así como la utilización de los desechos para la elaboración de alimentos balanceados, que conlleven a propiciar creación de empresas dedicadas desde la producción de la alcachofa hasta su transformación y comercialización, para satisfacer el mercado interno y para la exportación, de tal forma que se genere una cadena de valor altamente rentable para las zonas agrícolas productoras de esta hortaliza, teniendo como objetivo la posibilidad de la industrialización en base al rendimiento económico que de ella se derive.

Palabras clave: Pasta de alcachofa, aprovechamiento integral, cadenas productivas.

INTEGRAL INDUSTRIALIZATION OF THE ARTICHOKE IN NUTRITIONAL PASTA AND FOR BALANCED FOOD

\section{ABSTRACT}

A study of descriptive and experimental type was made, with the intention of processing the artichoke for the paste obtaining, as much for the human feeding, as for medicinal use and articles of beauty, as well as the use of the remainders for the balanced food elaboration, that entail to cause creation of companies dedicated from the production of the artichoke to their transformation and commercialization, to satisfy the internal market and for the export, of such form that is generated a chain of highly profitable value for the producing agricultural zones of this vegetable. Taking the possibility of the industrialization as an lens on the basis of the economic performance that from it stems.

Keywords: Paste of artichoke, integral advantage productive chains.

\section{INTRODUCCIÓN}

En el Perú, el proceso de industrialización de la alcachofa surge en la década de los 90 , cuando sale por primera vez al mercado los fondos de alcachofa en frascos, sea en salmuera o en aceite de oliva. Ahora, también se puede encontrar la alcachofa en picadillo, mas no en pasta se puede obtener la harina, el polvo micro pulverizado y de la utilización de los desechos se puede elaborar extractos y complementos para alimentos balanceados.

El desarrollo de la presente investigación ha permitido conocer la posibilidad de la industrialización integral de la alcachofa; y de realizarse a escala industrial, se lograría cerrar la cadena productiva que conlleva a dinamizar y ampliar el cultivo de alcachofa incrementar el número de empresas en la actividad agroindustrial y comercializar a nivel nacional e internacional los productos obtenidos.

Vale señalar que el objetivo de la investigación está encaminado a demostrar la viabilidad de industrializar integralmente la alcachofa, tanto su pulpa, tallo y hojas.

\section{JUSTIFICACIÓN}

Al procesar hortalizas que se encuentran actualmente en el mercado, permitirá mejorar la cadena productiva de estas, conllevando a propiciar la creación de empresas dedicadas en los diferentes rubros, desde la producción de la alcachofa hasta su transformación y comercialización, para satisfacer el mercado local e internacional.

Por otro lado, se ha tomado en consideración que los consumidores de hoy son mucho más exigentes que antes y que cada vez más demandan alimentos fáciles de preparar pero manteniendo una alimentación natural y con alto valor nutritivo, por lo que se justifica que este proyecto sea de utilidad tanto para las empresas como para los directos consumidores de alcachofa, lo que hará que la demanda interna se incremente, y con ello se cierre una cadena de valor altamente rentable.

La tendencia de los hábitos de consumo demandan que los productos sean inocuos, prácticos, innovativos y competitivos, estas condiciones hacen de la pasta de alcachofa, el extracto y el alimento balanceado insumos con grandes posibilidades de posicionamiento en el consumidor final.

(1) Docente de la Facultad de Ingeniería Industrial, Magíster en Promoción del Desarrollo y en Gestión y Administración teogaza57@yahoo.es 
Otra de las razones por las que se justifica el desarrollo de la investigación es el de facilitar el consumo de alcachofa a todos los usuarios, tanto al consumidor directo como a las diversas industrias tales como:

La industria panificadora. Que requiere de la alcachofa trozada y en pasta para la elaboración de empanadas, bocaditos, y otras variedades.

La industria farmoquímica. Que requiere la pasta de alcachofa para la elaboración de cremas de belleza, tanto para la piel como para el tratamiento del cabello.

La industria de medicina natural. Que requiere de harina de alcachofa para desayunos instantáneos, y para encapsularla, se requiere de la alcachofa micro pulverizada y del tallo y desechos blandos semi procesados para la preparación de extractos y de las hojas para la preparación de filtrantes.

La industria de alimentos balanceados. Requiere de la parte blanda y desechos del corazón y tallo de alcachofa para el fortalecimiento del forraje.

En la actividad gastronómica. Los restaurantes y las empresas dedicadas a la preparación de bufetes, presentarían diferentes platos y bocaditos en base a alcachofa si se le alcanzara ya trozada y en pasta.

\section{OBJETIVO GENERAL}

Procesar la alcachofa en pasta, para la alimentación humana, uso medicinal, artículos de belleza y de los desechos para la elaboración de alimentos balanceados.

\section{OBJETIVOS ESPECÍFICOS}

Elaborar el Balance de Materia, donde se establezca el volumen de materia prima para producción de pasta según la formulación del producto y la cantidad de desechos para la producción de extractos y la fibra para alimentos balanceados.

Calcular el rendimiento del proceso y el costo del producto final.

\section{HIPÓTESIS}

Al procesar la alcachofa en pasta para ser utilizada en la alimentación humana, uso medicinal, artículos de belleza, así como los desechos para la elaboración de alimentos balanceados, se facilitará el consumo y por consiguiente se incrementará la demanda, se generará mayor valor agregado y se promoverá la implementación de nuevas empresas en el rubro alimentario.

\section{MARCO TEÓRICO}

\section{Origen}

La Alcachofa (Cynara escolymus) es una planta herbácea originaria de la región del Mediterráneo (Asia menor y norte de África) y el sur de Europa. Parece ser que el cultivo y selección fue durante la Edad Media, quedando las mejores en España e Italia. De Italia fue introducida a Inglaterra, en 1548, y a los Estados Unidos, en 1806, siendo el Estado de California el principal productor.

En el hemisferio sur, solo figuran como productores Argentina, Chile y el Perú que ha pasado de tener un área muy pequeña a un área mayor en año 2003.

La palabra alcachofa parece que se deriva de un término árabe que significa "lengüetas de la tierra", en referencia a sus singulares hojas. Fueron los árabes, durante la Edad Media, quienes extendieron el cultivo por Europa, mejoraron las variedades y sus cualidades gastronómicas.

\section{Valor nutritivo}

Debido a su bajo contenido calórico y alta proporción de fibra, la alcachofa puede ser considerada una hortaliza light. El valor nutritivo aproximado de una alcachofa con una porción comestible de 100 gr. de corazón (fondo más las hojuelas interiores despuntadas) se muestra en el cuadro N. ${ }^{\circ} 1$.

\section{CARACTERÍSTICAS}

Forma. La parte comestible es la inflorescencia, alargada o achatada, en forma de rosetón, con hojas verdes superpuestas que parecen escamas y unidas al vástago. A las brácteas blandas internas y las porciones blandas del cogollo se les llama de manera coloquial "corazón de la alcachofa", y el fondo es la base carnosa.

Tamaño y peso. El tallo es erguido, grueso, acanalado longitudinalmente y ramificado. Llega al metro de altura y se divide en ramas que llevan en el extremo grandes inflorescencias de unos doce 


\begin{tabular}{|l|l|l|l|}
\hline \multicolumn{4}{|c|}{ Cuadro N. 1. Valor nutritivo de la alcachofa } \\
\hline COMPUESTOS & CONTENIDO & COMPUESTOS & CONTENIDO \\
Agua & $86,5 \%$ & Calcio & $51 \mathrm{mgr}$. \\
Ceniza & $1,8 \mathrm{gr}$. & Magnesio & $10 \mathrm{mgr}$. \\
Calorías totales & 4,0 & Fósforo & $69 \mathrm{mgr}$. \\
Calorías de grasa & 0 & Potasio & $310 \mathrm{mgr}$. \\
Carbohidratos & $9,9 \%$ & Hierro & $1,1 \mathrm{mgr}$. \\
Azúcares & $1,0 \mathrm{gr}$. & Ac. Ascórbico (Vit. C) & $8,0 \mathrm{mgr}$. \\
Proteínas & $2,8 \mathrm{gr}$. & Tiamina (Vit. B1) & $0,07 \mathrm{mgr}$. \\
Grasas & $0,2 \mathrm{gr}$. & Riboflavina (Vit. B2) & $0,04 \mathrm{mgr}$. \\
Fibra & $3,4 \mathrm{gr}$. & Niacina & $0,85 \mathrm{mgr}$. \\
Colesterol & 0 & Retinol (Vit. A) & $150 \mathrm{mgr}$. \\
\hline
\end{tabular}

Fuente: MINAG- INIA.

centímetros de diámetro cuando alcanzan su mejor tamaño. El peso del fondo varía según la variedad y el uso culinario, desde 50 gr hasta unos 100 gr.

Color. Hojas y tallo de color verde claro brillante cuando está recién cosechada.

Sabor. La pulpa de la alcachofa es crujiente (si se toma asada), suculenta, jugosa, fina, con una combinación muy acertada de sabores, dotada de un ligero amargor y un toque final dulce.

Propiedades medicinales. La alcachofa ha sido bastante estudiada por biólogos y médicos naturistas, por ello se puede relatar las diferentes propiedades medicinales y motivo por el cual esta investigación se apoya para su industrialización; es así que, entre otras propiedades, se distinguen las siguientes:

El potasio, debido a su alto contenido, es un mineral necesario para la transmisión y generación del impulso nervioso y para la actividad muscular normal.

\begin{tabular}{|l|l|}
\hline & Cuadro N. ${ }^{\circ} 2$ \\
\hline & \\
Leyenda: \\
1. Brácteas \\
2. Corazón \\
3. Fondo \\
4. Tallo
\end{tabular}

Fuente: MINAG- INIA.
El magnesio se relaciona con el funcionamiento del intestino, nervios y músculos; forma parte de huesos y dientes, mejora la inmunidad y posee un suave efecto laxante. La fibra, abundante en las alcachofas, favorece el tránsito intestinal. La inulina es un polisacárido que sustituye al almidón (reserva de moléculas de glucosa en los vegetales) y que también tiene función de reserva (unidades de fructosa en lugar de glucosa). Lo más destacable de la composición de la alcachofa son una serie de sustancias que no destacan por su cantidad, pero sí por los notables efectos fisiológicos que provocan tales como: La cinarina y cinaropicrina: compuestos aromáticos que dan el sabor amargo de la alcachofa. La cinarina se conoce por su efecto colerético y diurético.

Líneas de investigación actuales se centran en el potencial papel preventivo de la cinaropicrina en enfermedades tumorales. Ácido clorogénico: Compuesto fenólico con capacidad antioxidante. Esteroles: Sustancias vegetales con semejanza química al colesterol animal, con capacidad para limitar la absorción del colesterol en el intestino. Cinarósido: Flavonoide de acción antiinflamatoria. Ácidos orgánicos (málico y cítrico, entre otros): Se sabe que potencian la acción de la cinarina y del cinarósido, entre otras muchas funciones.

La alcachofa ha sido el prototipo de hortaliza sana, de amplio espectro medicinal. Se ha recomendado habitualmente en el campo de la nutrición por considerar que su consumo es muy saludable a distintos niveles del organismo, gracias a sus reconocidas propiedades coleréticas, hepatoprotectoras y diuréticas.

Favorece el crecimiento y desarrollo de los niños y el esfuerzo físico de los deportistas favorecen la función hepática, y de la vesícula biliar, con lo que me- 
jora la digestión. La fibra tiene capacidad de absorber agua, aumenta el volumen de las heces, de manera que ayuda a corregir el estreñimiento.

Además, la fibra proporciona sensación de plenitud, lo que conduce a la persona a ingerir menos alimentos. Reduce los niveles de colesterol, evita elevaciones bruscas de la glucemia por eso es saludable para los diabéticos, provoca un aumento de la cantidad de orina eliminada. Esto resulta beneficioso en el caso de sufrir cálculos renales, hiperuricemia, hipertensión arterial, retención de líquidos u oliguria (producción escasa de orina).

Los extractos de los tejidos finos de la alcachofa se utilizan en cosmética como ingrediente de los tónicos o lociones ligeramente astringentes útiles para limpiar y refrescar el cutis o para vigorizar el cabello y las hojas de la alcachofa se utilizan para fabricar un licor llamado Cynar, que se usa como aperitivo amargo o "bitter".

\section{LOCALIZACIÓN DE LA PRODUCCIÓN AGRÍCOLA DE ALCACHOFA EN EL PERÚ (año 2005-2006)}

Según la fuente del Ministerio de Agricultura (MINAG), Dirección General de investigación Agraria y Dirección General de Planificación Agraria (DGIA-DGPA), los Valles que destacan en cuanto a hectáreas cultivadas, el $72 \%$ de la producción de alcachofa se concentra en la Costa, siendo La Libertad, Lima, Áncash e Ica las de mayor concentración; mientras que en la Sierra, este cultivo se produce en Junín y Apurímac (ver cuadro N. 3 ).

Hace unos 10 años, la superficie cosechada de alcachofa fue de 200 ha con una producción de 1881 tm.; al año 2003 se incrementó a 2430 ha, con una producción de 23308 tm; mientras que el año 2005, aumentaron a 3175 ha. ADEX reportó que en condiciones favorables, durante el año 2006, la superficie sembrada fue, aproximadamente, de 8000 ha.

El nivel de rendimiento promedio por hectárea al año 2005, fue de 15,30 tm/ha; estos niveles de rendimiento promedio nacional ubica al Perú entre uno de los principales países con rendimientos superiores a otros países del mundo, ubicándose en el tercer lugar después de Egipto y Estados Unidos. (ver cuadro N. ${ }^{\circ} 4$ ); de acuerdo a las proyecciones de PROMPEX, se estima que al año 2006, la productividad mejorará esperando una cosecha en promedio de $18 \mathrm{tm} / \mathrm{ha}$, (ver cuadro N. ${ }^{\circ}$ ).

En cuanto a la cosecha, se puede comparar al Perú con los principales países productores de Europa, Estados Unidos y Latinoamérica, donde la estacionalidad es muy marcada; mientras que en el Perú las cosechas se realizan durante todo el año, como puede apreciarse en el cuadro $\mathrm{N} .^{\circ} 6$, en el resto de países es bien marcada la estacionalidad, este hecho le da competitividad a nuestro producto, puesto que la demanda se incrementa en los meses donde no hay producción en los otros países.

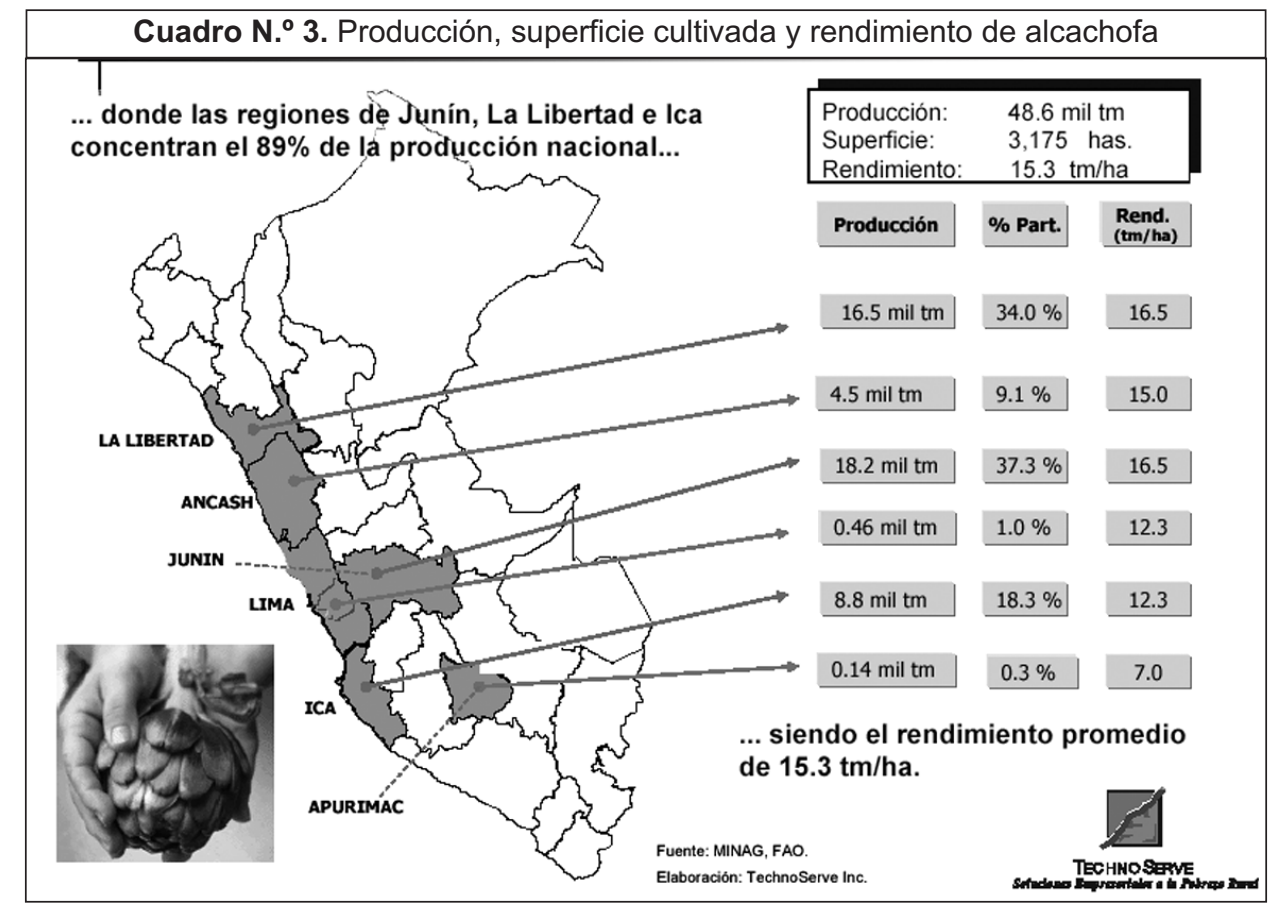

Fuente: FAO-MINAG, Elaboración: TechnoServe Inc 


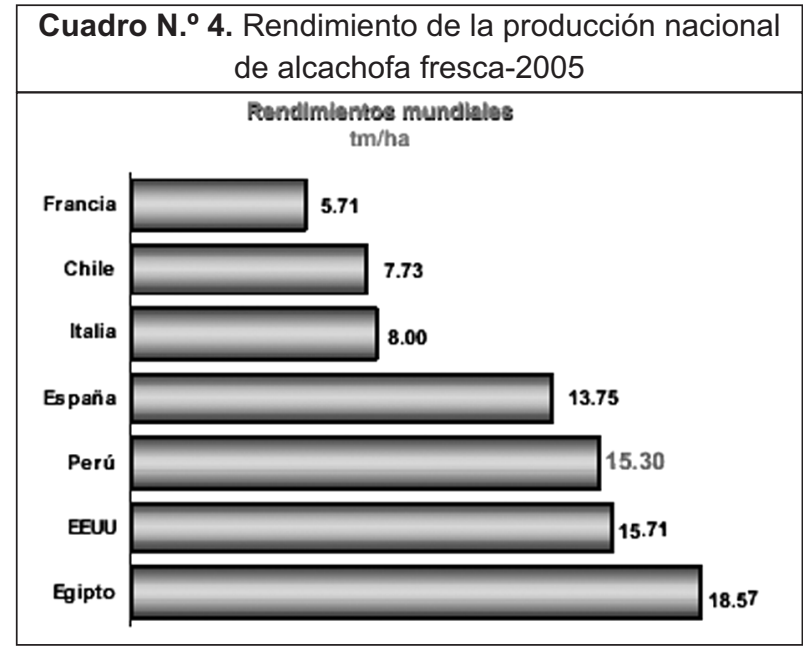

Fuente: FAO-MINAG.

Elaboración: TechnoServe Inc.

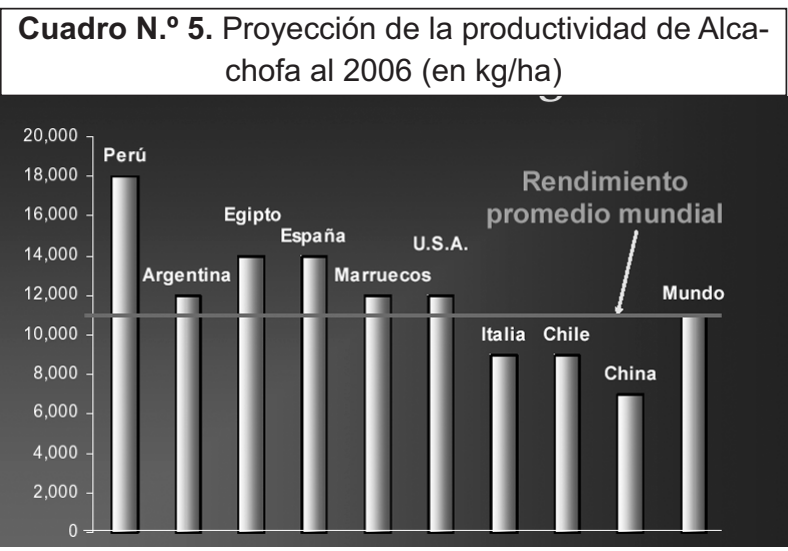

Fuente: PROMPEX.

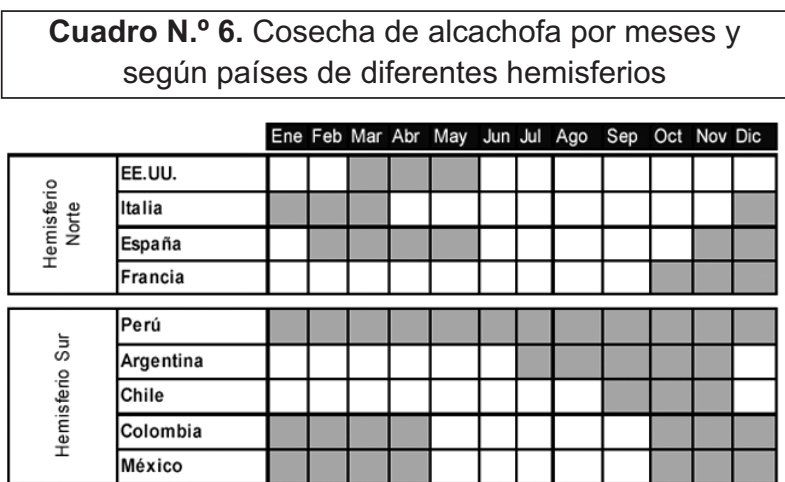

Fuente: MINAG-PROMPEX.

Elaboración: TechnoServe Inc.

\section{PROCESAMIENTO DE LA ALCACHOFA PARA LA OBTENCIÓN DE LA PASTA, EXTRACTO Y ALI- MENTO BALANCEADO}

Para el inicio del procesamiento de la alcachofa, tanto para la obtención de las pasta como para la obtención de los alimentos balanceados, se inicia con la separación del tallo y las hojas hasta encontrar el corazón mismo de la alcachofa, a este proceso se le denomina pelado; es en este momento que se hace la separación de las hojas de la parte blanda y el corazón, debido a que cada una de estas partes tiene un proceso diferente; es así que de la pulpa (fondo) se obtiene la pasta, del corazón, de las hojas y el tallo se obtiene el extracto y luego de este desecho se obtiene la fibra espesa que combinada con los desechos de la parte blanda se obtiene la mezcla espesa para los alimentos balanceados.

Para la obtención del pelado, los europeos, sobre todo los españoles, tienen diferentes máquinas. Para el presente caso de investigación, solamente se ha tomado como referencia para la comparación con el pelado manual.

\section{Características Generales}

- Máquina a movimiento continuo tipo circular con estaciones múltiples de alimentación, apta para alcachofa cocida o alcachofa en fresco alimentada para 120 unidades por minuto.

- La máquina se alimenta manualmente en forma continua, mientras las operaciones para obtener los corazones son comandadas por un sistema de camas especiales, sincronizadas con la rotación continua de máquina, con consiguiente notable aumento de la producción respecto a máquina de tipo alternativo.

- Colector distribuidor conductores de agua en tubos de 4x6 mm, llaves de regulación para la limpieza de cuchillas circulares de corte de tallo y hoja y cuchillas de corte de torneado.

- Cuadro de mandos en poliéster con baliza de indicación del funcionamiento y averías. El cuadrado tiene un sinóptico con indicaciones luminosas del funcionamiento de todos los motores de la máquina, igualmente indica la situación del sistema de engrase y del cambio del formato cuando está llevándose a cabo.

\section{Las operaciones que realiza la máquina en se- cuencia automática son:}

1. Corte inferior del tallo.

2. Posicionamiento automático de la alcachofa.

3. Torneado de dos alcachofas a la vez.

4. Corte superior, despunte de hojas.

5. Descarga de tallos, despunte y limpieza superficial de las hojas ya separadas de los corazones.

6. Expulsión de corazones y residuos de alcachofa. 
La máquina dispone de todas las medidas de seguridad relativas a la ley de seguridad en máquinas (CE) y sus correspondientes directivas, sensor de seguridad en zona de torneado y protección de seguridad en zona de torneado, así como en todas las partes móviles accesibles. Los materiales utilizados para la construcción de la máquina son: $A / I N O X A I S I$ 304, BRONCE, ALUMINIOS Y PLÁSTICO ATÓ$\mathrm{XICO}$.

Si se quiere aminorar los costos de este primer proceso, se debería adquirir una peladora de alcachofa, una muestra de esta máquina con sus características técnicas se puede apreciar en la Figura $N .^{\circ} 1$.

Sin embargo, es preciso señalar que el costo de la mano de obra en el Perú es mucho mas barata, por lo que se puede dar empleo a mucha gente del lugar. Considerando que cuando la mano de obra de especialización es normal, estas personas logran procesar en una jornada de $8 \mathrm{hrs}$ hasta $80 \mathrm{~kg}$ de alcachofa. Sin embargo, se ha podido observar que hay personas altamente especializadas que logran obtener en el mismo tiempo hasta $100 \mathrm{Kg}$ de alcachofa.

\section{PROCESAMIENTO DE LA ALCACHOFA EN PASTA}

\section{CÓMO ELEGIRLA Y CONSERVARLA}

A la hora de comprar alcachofas, hay que seleccionar las más gordas y pesadas en proporción a su tamaño, con las yemas gruesas, compactas, bien formadas y de color verde claro. El tamaño no afecta la calidad de la alcachofa.

Para comprobar su frescura, se puede apretar la alcachofa cerca del oído; si se escucha un crujido, aún está fresca. Si las hojas están blandas en la base o se abren con facilidad y presentan partes pardas, la alcachofa no está fresca, el fondo se ha secado y endurecido.

El frío hace que las hojas externas tiendan a ampollarse y adquirir primero un aspecto blanquecino que luego se vuelve marrón parduzco. Esta es la razón por la que las denominadas "alcachofas de invierno" pueden presentar manchas externas que hacen dudar de su frescura. Sin embargo, el aspecto no afecta la calidad culinaria del fruto.

Tras la cosecha, las alcachofas se han de almacenar en lugares frescos lo antes posible. Si se introducen en una bolsa de plástico cerrada y se dejan en el frigorífico, se conservarán una semana. Con tallo, se puede mantener en agua, como una flor cortada, en el frigorífico. Para que no se seque, lo mejor es no cortar el tallo hasta el momento de su consumo. Una vez cocidas, se han de conservar en el frigorífico y consumir en 24 horas. También se pueden congelar para aumentar su conservación durante varios meses.

\section{DESCRIPCIÓN DEL PROCESO}

Elaboración: (CAVIA, 2005) La clave para preparar una buena pasta reside en lavar y pelar debidamente la alcachofa. El siguiente aspecto a tener en cuenta en el momento de la preparación consiste en quitar las hebras, semillas, y demás sobrantes. Tras estos primeros pasos básicos, lo aconsejable es cocer los ingredientes para aprovechar al máximo su sabor, y lo que es más importante, conservar sus propiedades nutritivas originarias. "Con el fin de evitar la pérdida de vitaminas y minerales, se ha demostrado que la mejor forma de cocer verduras es no trocearlas mucho, con poca agua y efectuarlo en olla a presión", o cocinarlas a vapor, Para evitar que las alcachofas crudas se oxiden y se oscurezcan mientras se están limpiando y preparando para su cocción, se debe frotarlas con zumo de limón.

Para cocerlas, se utilizará un recipiente de acero inoxidable o de barro con agua suficiente que las cubra y, disuelta en esa agua, una cucharada de aceite de oliva y una pizca de sal (las alcachofas se oscurecen si se hierven en un recipiente de aluminio o quedan expuestas al aire durante la cocción). El aceite que se añade al agua hirviendo también evita que queden expuestas al oxígeno y tomen color desagradable.

De esta manera, el caldo de la cocción de las alcachofas se puede utilizar como si fuese una infusión o para el preparado de la misma pasta al licuarla.

Tras la cocción, el siguiente paso es el de la trituración. Ésta depende en gran medida del método que se utilice: el pasapurés, cuyo resultado es más grumoso, o la batidora, con la que se obtiene una textura más suave.

\section{OBTENCIÓN DEL EXTRACTO Y ALIMENTO BLANCEADO}

- Hervir a una temperatura de $80^{\circ} \mathrm{C}$ las hojas, tallo y la parte blanda de los desechos de la 
alcachofa por, aproximadamente, una hora a fuego medianamente fuerte hasta que las hojas y el tallo estén bastante blandas.

- Licuar regularmente toda esta mezcla hasta que se pueda observar la separación del líquido y la fibra.

- Colar y obtener así el extracto que servirá para el consumo humano (si uno lo desea) y separar la parte fibrosa que servirá para el alimento balanceado de animales menores (patos, cuyes, conejos, etc.) o como complemento del forraje para la ganadería lechera. Para este caso, es recomendable usarlo como insumo en la mezcla con otros que forman parte de la dieta alimenticia del ganado, combinándolo adecuadamente para la ración diaria del ganado vacuno. (ALATA, 2006)

\section{BALANCE DE MATERIA PRIMA Y PRODUCTO TERMINADO}

Antes de dar inicio al balance de materia prima empleada para el experimento motivo del proyecto, es preciso señalar la diferencia de costos existentes, tanto en chacra como en mercados mayorista y minorista, de tal forma que se pueda, en una segunda parte del proyecto, elaborar este balance con mayor precisión, y demostrar cuánto más estarían ganando los agricultores si en sus mismos lugares de producción de alcachofa la procesasen para satisfacer la demanda de los usuarios nacionales e internacionales.

Se ha podido identificar los siguientes precios al 3010-07:

\begin{tabular}{|c|c|c|c|}
\hline \multicolumn{4}{|c|}{$\begin{array}{c}\text { Cuadro N. }{ }^{\circ} \text { 7. Precios por kilogramo y llevados a canti- } \\
\text { dades unitarias de la alcachofa }\end{array}$} \\
\hline Alcachofa & $\begin{array}{l}\text { Alcachofa } \\
\text { Cantidad }\end{array}$ & $\begin{array}{l}\text { Unidad de } \\
\text { medida (Kg) }\end{array}$ & $\begin{array}{l}\text { Precio en } \\
\text { soles (S/.) }\end{array}$ \\
\hline \multirow[t]{2}{*}{ Chacra } & 3 & 1 & 1,20 \\
\hline & 12 & 1 fondos & 4,80 \\
\hline Mercado & 3 & 1 & 2,00 \\
\hline Mayorista & 12 & 1 fondos & 8,00 \\
\hline Verdulerías & 3 & 1 & 3,00 \\
\hline al por menor & 12 & 1 fondos & 10,00 \\
\hline
\end{tabular}

Fuente: Elaboración propia.
Como puede apreciarse las diferencias de precios son elevadas entre el precio en chacra, mayorista y minoristas, dentro de esta cadena de comercialización. La frescura de los frutos también hace que varíe los precios y, por ende, la calidad del producto, por todo lo que significa el tiempo de traslado hasta llegar al consumidor final.

Este hecho nos hace reflexionar sobre la importancia que tiene el que las empresas procesadoras de alcachofa estén ubicadas en el mismo lugar de los cultivos, ya que la competitividad se eleva frente a otros lugares de localización. Además, se tendrían mayores ventajas económicas para el agricultor y la gente del área rural, por la cantidad de desechos que quedan en la zona de cultivos después de la cosecha, que pueden ser utilizados.

\section{BALANCE DE MATERIA PRIMA Y PRODUCTO TERMINADO}

Se tomó como base referencial un kilo de alcachofa y se observó que tres unidades de alcachofas frescas equivalen a un kg, obteniéndose los siguientes resultados:

- 250 gr de fondos

- 300 gr de corazones

- 450 gr de hojas y tallos

Observando el diagrama $\mathrm{N}^{\circ} 1$, se puede apreciar los ingresos de materia prima y los resultados en producto terminado:

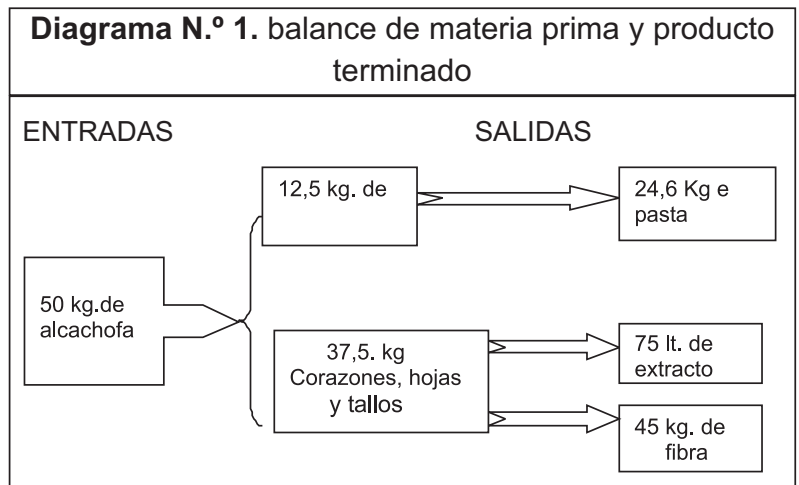

Fuente: Elaboración propia.

Con estas cantidades se desarrolló el experimento para la obtención de pasta y extracto, orientada directamente para el consumo humano y la parte de fibra para la formación del alimento balanceado. 
ANÁLISIS DE COSTOS DE PRODUCCIÓN PARA EL PROCESAMIENTO DE LA PASTA DE ALCACHOFA, EXTRACTO Y FIBRA PARA ALIMENTOS BALANCEADOS (en una jornada de 8 hrs.)

\begin{tabular}{|c|c|c|c|c|}
\hline CONCEPTOS & CANTIDAD & $\begin{array}{l}\text { UNIDAD DE } \\
\text { MEDIDA }\end{array}$ & $\begin{array}{l}\text { PRECIO } \\
\text { UNITARIO } \\
\text { S/. }\end{array}$ & $\begin{array}{l}\text { PRECIO } \\
\text { TOTAL } \\
\text { SI. }\end{array}$ \\
\hline \multicolumn{5}{|l|}{ MATERIA PRIMA } \\
\hline Directa (alcachofa) & 50 & $\mathrm{~kg}$ & 8.00 & 400.00 \\
\hline Indirecta (insumos) & & & 30.00 & 30.00 \\
\hline \multicolumn{5}{|l|}{ MANO DE OBRA } \\
\hline Mano de obra directa & 1 & persona & חמת מכ & ח 20 \\
\hline \multicolumn{5}{|l|}{ CARGOS INDIRECTOS } \\
\hline Gastos administrativo & & servicios & 10.00 & 10.00 \\
\hline \multicolumn{5}{|l|}{ Artículos para el } \\
\hline Limpieza & & bienes & 20.00 & 20.00 \\
\hline COSTO TOTAL DE P & ODUCCIÓN & & & \\
\hline
\end{tabular}

Considerando:

- Utilidad deseada del $15 \%$

- $50 \mathrm{~kg}$ de alcachofa

- 1 jornada de trabajo

- 1 persona

- Uso de máquinas y equipamiento doméstico

- Luego del procesamiento se obtienen los siguientes resultados:

\begin{tabular}{llcc|}
\hline Producto terminado & cantidad & Precio unitario (S/.) & Total $(\mathbf{S} /)$. \\
\hline Pasta de alcachofa & $24,60 \mathrm{Kg}$ & 22,44 & 552,00 \\
Extracto & $75 \mathrm{lt}$. & 2,00 & 150,00 \\
Fibra & $45 \mathrm{~kg}$. & 1,00 & 45,00 \\
\hline TOTAL DE INGRESOS POR PRECIO DE VENTA & $\mathbf{7 4 7 , 0 0}$ \\
\hline
\end{tabular}

RESUMEN DEL BALANCE

$\begin{array}{lc}\text { CONCEPTOS } & \text { S/. } \\ \text { Ingresos } & 747,00 \\ \text { Egresos } & 480,00 \\ & \\ \text { Utilidad bruta } & 267,00\end{array}$

Como puede apreciarse de los resultados esperados con un $15 \%$ de utilidades deseables, solamente en el precio de la pasta, se ha recuperado la inversión; y utilizando los corazones, hojas y tallos en extractos y fibra, se incrementan los ingresos; con lo que queda demostrada la importancia de la utilización integral de la alcachofa.

Si esta experiencia se llevara a niveles industriales y en las mismas zonas de producción, sería un éxito para los agricultores y para el país, teniendo en cuenta las economías de escala y los costos de la alcachofa en chacra.

En esta primera etapa del proyecto, la elaboración fue artesanal, debido a que la maquinaria automatizada que se está montando en el laboratorio de la Facultad de Ingeniería Industrial, aún no está disponible para los ensayos integrales que nos permitan tener una idea de los costos que se derivarían al utilizarla. 


\section{CONCLUSIONES}

1. La alcachofa es una hortaliza reconocida por los biólogos y médicos como una especie importante en la alimentación, tanto por sus propiedades medicinales como por su riqueza vitamínica para una dieta balanceada, que procesada, facilitaría su consumo y se convertiría en un nuevo producto que el Perú puede explotar para incursionar en las exportaciones de productos alimenticios procesados

2. El Perú, por su estacionalidad y condiciones climáticas, es uno de los países que cosecha todo el año, razones por las cuales lo hacen competitivo; siendo los meses de diciembre, enero, febrero, julio, agosto y septiembre las mejores épocas para llegar al más grande mercado que es Estados Unidos de Norteamérica

3. Mientras dure el tratado de la ATPDEA, que permite el acceso al mercado de Estados Unidos con arancel cero para la alcachofa, ya sea procesada o ya sea fresca, será una importante oportunidad para los agricultores y pequeños empresarios del sector agroindustrial del Perú.

4. Los nuevos hábitos de consumo de comida natural dentro de la dieta principal de los grandes mercados de Estados Unidos, Europa y Asia, abren nuevas posibilidades para la alcachofa, al presentarla tanto en pasta, como en extractos, cremas, en harinas, encurtidos marinados, néctar, cremas deshidratadas corazones y fondos congelados, puré, harina para fideos, cápsulas y comprimidos, el cultivo de alcachofa traerá muchos beneficios a los agricultores y pequeños empresarios del sector agroindustrial y a la exportación en el Perú.

5. Los rendimientos económicos producto del procesamiento realizado a nivel artesanal o doméstico, dan muchas esperanzas para la constitución de buenos negocios, sobre todo si estos se localizan en las inmediaciones de los cultivos de las zonas donde se produce la alcachofa, puesto que los costos de transporte y la cantidad de desechos abaratarían los costos de producción.

\section{RECOMENDACIONES}

1. Por la viabilidad técnica, económica y alimenticia del proyecto, así como generadora de valor agregado, es recomendable que se le considere dentro de los proyecto concursables por los fondos del BID, o hacia otras entidades, como Sierra Exportadora, para lo cual la Facultad debe impulsar y dar las facilidades necesarias a los docentes responsables, a fin de proponerlo ante las entidades correspondientes para su elegibilidad.

2. Por la reducida cantidad de dinero asignada al proyecto, no ha sido posible hacer un experimento a niveles industriales; aún si se tuviera que alquilar los servicios de otros laboratorios y plantas pilotos para su procesamiento, el monto asignado no alcanzaría, por lo que se recomienda, de estar interesada la facultad, poner en marcha una planta piloto de uso integral para hortalizas debe realizar el esfuerzo económico necesario o los contactos con la Cooperación Técnica Internacional para solicitar los equipos y maquinarias identificadas para este fin.

3. Se recomienda continuar con el proyecto para elevarlo a nivel de factibilidad con la participación de los agricultores de los distintos valles donde se produce la alcachofa.

4. El tratamiento para la conservación de este tipo de alimentos que se pretenden conservar con preservantes naturales estarían orientados a la utilización de la nueva metodología de radiaciones nucleares descubierta últimamente por el IPEN.

5. Las pruebas de la demanda creciente de alimentos seguros como resultado del comercio internacional y la globalización, lleva a la industria de procesamiento de alimentos a implementar el sistema de gestión de seguridad de alimentos basado en HACCP (Hazard Analysis and Critical Control Point) -ISO 22000-, cuyos costos son elevados pero necesarios para competir a nivel internacional; sin esta prueba, es imposible la permanencia en el mercado global de alimentos, por eso es recomendable trabajar con esta normatividad. 


\section{BIBLIOGRAFÍA}

1. ADUANA (2007). http://www.aduanet.gob.pe/alcachofas-exportaciones (visitado en septiembre de 2007).

2. Alata Olivares, N.S. (2007). Asesor empresarial, asesor técnico en ganadería intensiva, cultivos de agro-exportación y gerencia agropecuaria-sistemas de riego por goteo-uso de rastrojos de agroindustria en ganadería. http://www.engormix.com/uso_alcachofa_como_forraje_forumsview8872.htm (Visitado en octubre de 2007).

3. Cárdenas, G. (2006). Alcachofa, cualidades y producción. Colección Mi Huerto, ediciones RI-
PALME, 2006, Lima-Perú, ISBN Nº 9972-84030-1

4. INIA (2007). El cultivo de alcachofa y páprika con fines de exportación. http://www.inia.gob.pe/eventos/evento033/ (Visitado en agosto de 2007).

5. Robles, F. La alcachofa, nueva alternativa para la agricultura Peruana. http://cdiserver.mbail.edu.pe/mbapage/cdi/BoletinesElectronicos/Estudios\%20de\%20mercado/alcachofaalternativa2 001.pdf (Visitado en agosto de 2007)

6. SUNAT, http://www.sunat.gob.pe/alcachofas (Visitado en julio de 2007). 